\title{
Characterization of Active Integrated Multicore Waveguides
}

\author{
David Benedicto, Juan Carlos Martín, Juan Antonio Vallés \\ Tecnologías Ópticas Láser (TOL) \\ Instituto de Investigación en Ingeniería de Aragón (I3A) \\ Universidad de Zaragoza, Mariano Esquillor s/n, 50018, Zaragoza, Spain. \\ Tel. +34-876 5534 48, e-mail: dbenedicto@unizar.es
}

\begin{abstract}
This study includes the experimental both passive and active characterization of multicore waveguides written in phosphate glass codoped with erbium and ytterbium, with the aim of designing and optimizing photonic devices based on this type of structures.
\end{abstract}

\section{Integrated multicore waveguides}

The irradiation of phosphate glasses co-doped with $\mathrm{Er}^{3+}$ and $\mathrm{Yb}^{3+}$ ions with femtosecond laser pulses allows to control the geometry and composition of the modified region by ionic migration and, thus, the inscription of integrated waveguides with high index contrast $\left(\Delta n>10^{-2}\right)$ and at the same time of the enrichment of active ions in the nucleus [1]. In this work, the characterization of multi-core guides (figure 1a) inscribed in this type of glasses is presented in order to fit the numerical simulations and the subsequent design and optimization of the devices.

These waveguides are very interesting in order to obtain large modal areas, as well as for signal multiplexing, either spatial when the cores are uncoupled or modal in the opposite case. In addition, the guides in phosphate glass show great potential for applications such as mode converters, gain spectrum equalizers, sensors and in the field of astrophotonics.

\section{Passive and active characterization}

First, the active and passive characterization of the individual waveguides has been carried out [2]. Next, the parameters obtained from the characterization process are used to compare the theoretical and experimental behavior of signals propagating in waveguides with several coupled nuclei, and where the theoretical simulations are based on solving the system of equations of the propagation of optical powers coupled to the system of differential equations for the variation of the population density of the ions of the rare earths in the different energy states [3]. In addition, in highindex-contrast structures the crosstalk between cores can be reduced and for some writing energie and scan speeds relative gain values of up to $19 \mathrm{~dB} / \mathrm{cm}$ can be reached with passive losses lower than $1 \mathrm{~dB} /$ $\mathrm{cm}$ in individual waveguides. Active measurements of gain as a function of the separation between cores (figure 1b) and of the excitation (input pump power) allow to adjust the model in order to design and optimize these structures for diverse applications.

\section{Conclusions}

The characterization procedure of active integrated multicore structures is presented. This procedure is based on passive and active measurements on 1-core waveguides. Then, the obtained characteristic parameters can be used for the design and optimization of promising multicore photonic structures.

\section{REFERENCES}

[1]. FERNANDEZ, T. Toney, et al. Ion migration assisted inscription of high refractive index contrast waveguides by femtosecond laser pulses in phosphate glass. Optics letters, 2013, vol. 38, no 24, p. 5248 5251.

[2]. VALLÉS, J. A., et al. Performance of ultrafast laser written active waveguides by rigorous modeling of optical gain measurements. Optical Materials Express, 2011, vol. 1, no 4, p. 564-575.

[3]. GILES, C. Randy; DESURVIRE, Emmanuel. Modeling erbium-doped fiber amplifiers. Journal of lightwave technology, 1991, vol. 9, no 2, p. 271-283. 
a)
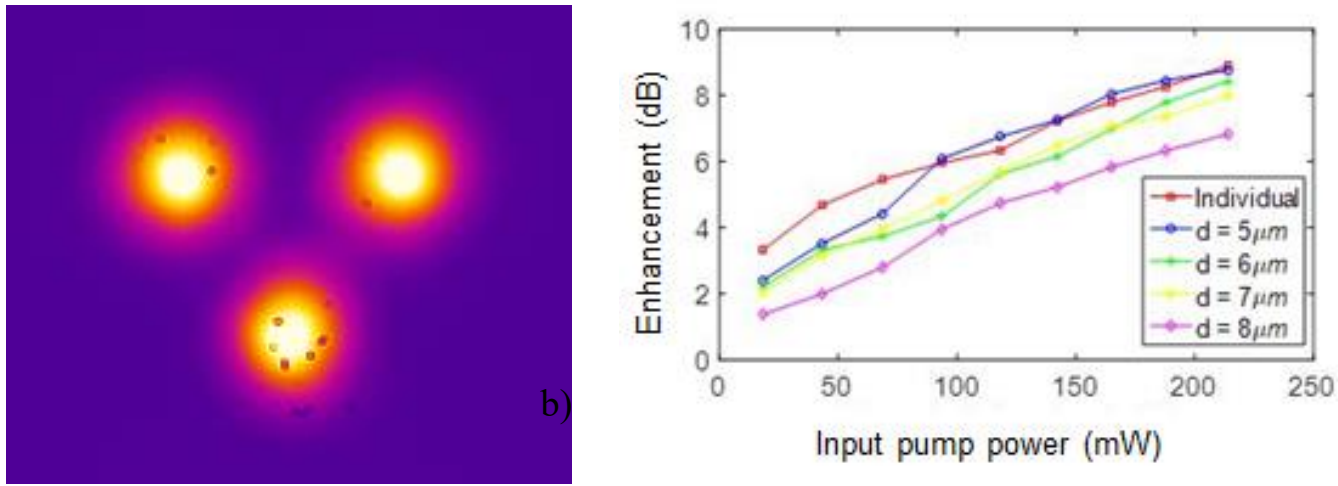

Figure 1 .- (a) Experimental measurement of the intensity distribution of a waveguide with three $5 \mu \mathrm{m}$-diameter cores separated $7 \mu \mathrm{m}$ between their centers for $\lambda=980 \mathrm{~nm}$ and (b) Enhancement (relative gain) as a function of pump power over a length of $1 \mathrm{~cm}$ for an individual waveguide and for 2-core waveguide with different separation between core centers. 